
https://doi.org/10.1007/s00739-020-00654-4 Online publiziert: 14. Juli 2020

(c) Der/die Autor(en) 2020 psychopraxis. neuropraxis $2020 \cdot 23: 175-179$

Felix Richter ${ }^{1}$ - Dagmar Steinmair ${ }^{1,2}$ - Henriette Löffler-Stastka'

' Klinik für Psychoanalyse und Psychotherapie, Medizinische Universität Wien, Wien, Österreich

${ }^{2}$ Klinische Abteilung für Augenheilkunde und Orbitachirurgie, Universitätsklinikum St. Pölten, St. Pölten, Österreich

\section{Mentalisierung bei Störungen aus dem schizophrenen Formenkreis}

\section{Fallorientierte Darstellung}

\section{Einleitung}

Der alltägliche zwischenmenschliche Kontakt ist davon geprägt, dass die Personen innerhalb von sozialen Interaktionen Gefühle und Gedanken des Gegenübers zu erschließen versuchen.

Unter Mentalisierung wird die Fähigkeit verstanden, mentale Zustände der eigenen Person wie auch dem Gegenüber zuschreiben zu können. Unter mentalen Zuständen werden Gefühle, Gedanken und Intentionen subsumiert. Es handelt sich folglich um eine imaginative Fähigkeit, den Zusammenhang von Verhalten und mentalen Zuständen sowohl wahrzunehmen als auch interpretieren zu können. In der wissenschaftlichen Literatur wird der Begriff Mentalisierung meist synonym für verschiedene Konzepte verwendet. Demnach kann das Konzept der Mentalisierungsfähigkeit als integratives Brückenkonzept verstanden werden, welches verschiedene Teilaspekte der sozialen Kognitionen vereint.

Eine Umgebung, welche die Entwicklung der Mentalisierungsfähigkeit fördert, ermöglicht es dem Kind, aufdas Verhalten anderer $\mathrm{zu}$ reagieren oder spezifischer: Die Mentalisierungsfähigkeit ermöglicht es dem Kind, auf die eigenen Vorstellungen bezüglich der anderen Personen $\mathrm{zu}$ reagieren und somit die Fähigkeiten oder Intentionen das Gegenübers zu verstehen. Dies hat zur Folge, dass die Stimmung erfasst werden kann und somit die Verhaltensweisen anderer Personen als bedeutungsvoll angesehen werden und ebenfalls Handlungen antizipiert werden können.

Die Reflexionsfunktion (RF) ermöglicht es dem Individuum, zwischen innerer und äußerer Realität zu unterscheiden. Diese individuelle Art der psychischen Realität wird von Fonagy und Kollegen als Modus psychischen Funktionierens beschrieben und lässt sich einteilen in den

- teleologischen Modus,

- den Modus der psychischen Äquivalenz,

- den Als-ob-Modus und den

- mentalisierenden Modus.

Diese sind hierarchisch angeordnet und folgen entwicklungspsychologisch aufeinander. Der Modus der psychischen Äquivalenz ist dadurch charakterisiert, dass das Individuum die innere Realität mit der äußeren gleichsetzt und somit das Verhalten maßgeblich vom subjektiven Erleben beeinflusst wird.

\section{Mentalisierungsfähigkeit - ein dynamischer Prozess}

In ihrer Konzeption der Mentalisierung fassen die Autoren die Mentalisierungsfähigkeit als dynamischen Prozess auf, welcher abhängig von Stress und Bindungssystem ist. Dieser Aspekt wird anhand des Stressmodells erklärt. Allen und Kollegen ergänzen Mayes' Ansatz dahingehend, dass sie das Arousal in $\mathrm{Zu}$ - sammenhang mit dem Bindungsstress und den jeweiligen individuellen $\mathrm{Be}$ wältigungsmustern setzen. Dementsprechend formuliert das erweiterte Modell das Umschalten von kortikalen $\mathrm{zu}$ subkortikalen Verarbeitungen anhand der Intensität des Bindungsstresses. Dies schränkt die Flexibilität der Mentalisierungsfähigkeit ein, sodass auf prämentalisierende Verarbeitungsmodi (Äquivalenz, Als-ob, teleologisch) zurückgegriffen wird. Innerhalb einer emotional aufgeladen Situation, wie einem partnerschaftlichen Streit, führt der erhöhte Bindungsstress dazu, dass auf die gewöhnliche Mentalisierungsfähigkeit nicht mehr zugegriffen werden kann und weniger integrierte Mentalisierungsmodi verwendet werden, sodass sich die Person nach der Situation über ihr Verhalten möglicherweise wundert oder dieses bereut. Der Schwellenwert kann dabei individuell variieren und steht in Abhängigkeit zum jeweiligen Bindungsmuster. Dementsprechend zeigen Individuen mit unsicher-verstricktem Bindungsmuster eine niedrigere Schwelle als sicher gebundene Personen.

\section{Mentalisieren bei Schizophrenie}

Christopher D. Frith entwarf ein kognitives Modell der Schizophrenie, anhand dessen er die Symptomatik psychologisch zu erklären versuchte. Kernbestandteil des symptomatischen Spektrums der Schizophrenie sei demnach die Unfähigkeit der Bildung von Metarepräsenta- 
tionen. Dieses Defizit hinsichtlich der Metarepräsentationen lässt sich nach Frith drei Domänen zuordnen, welche mit verschiedenen Facetten der psychotischen Symptomatik zusammenhängen.

Die erste Domäne ist die Störung der Handlungs- oder Entscheidungsstärke („disorders of willed action“). Demnach führt die Unfähigkeit, spontane und gewollte Handlungen durchzuführen, zu Negativ-Symptomen.

Die Störung des Self-Monitorings zeichnet sich dadurch aus, dass Patienten nicht in der Lage sind, ihre eigenen Intentionen wahrzunehmen und somit ihre Gedanken nicht als von sich selbst produziert wahrnehmen. Dies hat zur Folge, dass ihre Handlungen als von außen gesteuert interpretiert werden und somit zu Fremdbeurteilungserlebnissen führen. Ähnlich hierzu werden die eigenen Gedanken oder die subvokale Sprache nicht als eigene, sondern als von außen kontrolliert angesehen. Des Weiteren führt Frith die Störung der Zuschreibung von Intentionen anderer an („disorders in monitoring the intentions of others"). Demnach ist für die Entstehung von paranoidem Wahn und Verfolgungswahn die inkorrekte Zuschreibung von Intentionen anderer ursächlich.

\section{》) Kinder mit frühkindlichem Autismus weisen ein Defizit in der Mentalisierungsfähigkeit auf}

Kinder mit frühkindlichem Autismus weisen ebenfalls ein erhebliches Defizit in der Mentalisierungsfähigkeit auf, wobei sie keine positiven Symptome zeigen. Frith argumentiert, dass dies mit entwicklungsbedingten Aspekten zusammenhängt. Während Kinder mit frühkindlichem Autismus seit der Geburt ein Mentalisierungsdefizit aufweisen und sich eine ToM somit nicht (oder kaum) entwickeln konnte, bricht die schizophrene Erkrankung typischerweise in der Adoleszenz aus, sodass eine generelle Fähigkeit zum Mentalisieren stattgefunden hat. Dementsprechend wird in der psychotischen Episode die Mentalisierung weiterhin durchgeführt, jedoch kommt es hierbei zu fehlerhaften Zuschreibungen.

Verschiedene Studien konnten Friths Theorie stützen. Patienten mit negativen Symptomen („disorder of willed action“) zeigen ein deutliches Mentalisierungsdefizit. Patienten mit Symptomen von Fremdbeeinflussung, Gedankeneingebung ("disorder of self-monitoring“) und in Remission verhielten sich unauffällig hinsichtlich ihrer Mentalisierungsfähigkeit. Hinsichtlich des Zusammenhangs zwischen paranoider Symptomatik und Mentalisierungsdefizit zeigten sich widersprüchliche Ergebnisse, indem Studien ein Defizit nahelegen oder widerlegen.

Je nach Symptomatik kann das Mentalisierungsdefizit variieren. Es zeigte sich etwa, dass Patienten mit Passivitätsphänomen, d.h. der Auffassung zu sein, nicht mehr die Kontrolle über die eigenen Gedanken zu haben, kein Defizit hinsichtlich des fremdorientierten Mentalisierens, sondern nur hinsichtlich des selbstorientierten Mentalisierens aufweisen.

\section{》) Als Hypermentalisieren wird eine Überattribution von Intentionen verstanden}

Sousa, Sellwood, Spray und Bentall untersuchten den Zusammenhang zwischen „internal source monitoring“ (iSM) und formalen Denkstörungen bei psychotischen Patienten unter emotionaler Belastung. Es zeigte sich, dass niedrigere iSM mit formalen Denkstörungen zusammenhängen. Die Autoren postulieren, dass psychotische Patienten v.a. bei negativen Affekten ein ausgeprägtes iSMDefizit aufweisen, welches wiederum zu vermehrten formalen Denkstörungen führt.

Die Bedeutung der Mentalisierungsfähigkeit für schizophrene Psychosen kann nicht nur in Hinblick auf das Vor- oder Nicht-Vorhandensein dieser Fähigkeit bezüglich verschiedener Erkrankungsphasen oder Symptomatiken diskutiert werden, sondern auch in Hinblick auf die Art wie schizophrene Patienten mentalisieren.
Abu-Akel und Bailey verstehen, teilweise konträr zu Friths Auffassung, die Positivsymptomatik als eine Art Hypermentalisierung, indem es bei schizophrenen Patienten zu einer übermäßigen $\mathrm{Zu}$ schreibung von Absichten kommt.

Insbesondere die paranoide Schizophrenie wird charakterisiert durch eine Art des Hypermentalisierens. Als Hypermentalisieren wird eine Überattribution von Intentionen verstanden. Eine Erklärung, warum schizophrene Patienten beim ToM-Test eine Hypermentalisierung zeigen, ist, dass die Stimuli zu viele mentale Repräsentationen bei psychotischen Patienten hervorrufen, sodass es aufgrund konkurrierender Repräsentationen zu einer größeren Beeinträchtigung während der ToM-Aufgaben kommt.

Diese Art des Mentalisierens kann jedoch nicht als universell für alle schizophrenen Psychosen geltend gemacht werden. Es zeigt sich etwa, dass schizophrene Patienten in einem ToM-Test, welcher das Verständnis eines Witzes beinhaltete, hypermentalisierten, indem in der Kontrollaufgabe, welche nicht mentalisierende Themen beinhaltete, den Cartoonfiguren negative Absichten zugeschrieben wurden, als auch hypomentalisierten, indem die Patienten weniger mentale Zuschreibungen äußerten als eine Kontrollgruppe und den Witz nicht verstehen konnten. Ursächlich für beide Arten der Mentalisierung könnte eine gestörte Fähigkeit hinsichtlich der Perspektivenübernahme sein. Diese Schwierigkeit, verschiedene Perspektiven innerhalb derselben Situation einzunehmen, führt zu der egozentrischen Projektion eigener Neigungen auf unbeteiligte andere (Hypermentalisieren) sowie der Unfähigkeit, komplexe soziale Situationen anhand verschiedener Perspektiven interpretieren zu können (Hypomentalisieren).

Sass und Parnas verstehen die Schizophrenie primär als eine Störung des Selbst, welche sich durch abnormales Bewusstsein und sich ergänzende Verzerrungen des Bewusstseins charakterisiert: Hyperreflexivität und verminderter Bezug zu sich selbst. Demnach ist Hyperreflexivität übertriebene Selbstwahrnehmung, wobei eigene Aspekte mit externalen Objekten verschmolzen sind. Der ver- 
minderte Bezug zu sich selbst wird verstanden als verminderte Fähigkeit, sich selbst als aktiver Akteur zu fühlen und als Quelle seiner eigenen Handlungen zu sehen.

Des Weiteren wurden die Mentalisierungsdefizite innerhalb der psychotischen Störungen mit der symptomatischen Ausprägung in Zusammenhang gesetzt. Demnach zeigen schizophrene Patienten Schwierigkeiten, Gedanken, welche vom Individuum produziert wurden, von externen produzierten Stimuli $\mathrm{zu}$ unterscheiden („source-monitoring "). Dies gilt insbesondere für Patienten mit akustischen Halluzinationen und Gedankeneingebung.

Psychotische Störungen gehen mit einer starken sozialen Beeinträchtigung einher, welche sich in einer Arbeitsunfähigkeit oder einer generell niedrigen Lebensqualität äußert. Diese soziale Beeinträchtigung könnte durch eingeschränkte Mentalisierungsfähigkeit vermittelt werden. In einer Metaanalyse von Fett et al. zeigten die Forscher, dass gerade die Mentalisierungsfähigkeit den stärksten Zusammenhang zur sozialen Dysfunktion aufweist und dass dieser Zusammenhang stärker war als bei Neurokognitionen und anderen sozialen Aspekten.

\section{Studie}

In einer eigens durchgeführten Studie konnten wir sowohl einen Zusammenhang zwischen einer niedrigen Mentalisierungsfähigkeit und Aufmerksamkeitsund Sprachbeeinträchtigungen (d.h. einer defizitären Wahrnehmung und Interpretation externer Stimuli) aufzeigen als auch, dass die Mentalisierungsfähigkeit in einem negativen Zusammenhang mit einem vermeidenden Bindungsmuster in partnerschaftlichen Beziehungen assoziiert ist.

Zusammenfassend lässt sich die Mentalisierungsfähigkeit von Patienten mit schizophreniformen Störungen wie folgt abbilden:

Die generelle Fähigkeit zu mentalisieren, scheint überdauernd vermindert im Vergleich zu gesunden Personen. In akuten Krankheitsphasen wird diese Mentalisierungsfähigkeit durch
(Bindungs-)Stress weiter vermindert. Dann zeichnen sich die Modi des psychischen Erlebens durch prämentalistische Erlebensmuster aus (teleologisch, Äquivalenzmodus). Es kommt zu einer gravierenden Hemmung des Mentalisierens (Hypomentalisieren) oder $\mathrm{zu}$ einer massiven Fehlzuschreibung von mentalen Prozessen (Hypermentalisieren). Dieser Zusammenbruch ist hierbei funktional zu verstehen, indem es dem Individuum als situativer Schutzmechanismus vor überwältigenden Gefühlen von Scham oder Trauma dient.

\section{Fallbericht}

Brent schildert den Fall von Victor, dessen Schizophrenie und die Behandlungserfolge im Einzelsetting mittels mentalisierungsbasierter Psychotherapie (MBT). Soziale Isolation und ein „depressives“ Zustandsbild hatten zur Vorstellung des 20-jährigen Patienten geführt, welcher sich selbst als „bad“, „damaged“ und als „outsider“ bezeichnete. Die Eltern wurden durch Victor als emotional unzugänglich beschrieben, die Vaterfigur idealisiert. Den Personen in seinem Umfeld misstraute Victor, da er ihnen unterstellte, ihn ohne Weiteres als „damaged“ wahrnehmen zu können. Die Fallschilderung besticht durch das klare Herausarbeiten der o.g. typischerweise bei schizophreniformen Störungen auftretenden verminderten Source-monitoring-Fähigkeit mit akustischen Halluzinationen (abwertende und kommentierende Stimmen), eingeschränkter Perspektivenübernahme und Hypermentalisierung im Sinne der $\mathrm{Zu}$ schreibung eigener (negativer) Gedanken, Gefühlszustände und Motive an das Umfeld. Brent arbeitet in der Fallvignette insbesondere Victors Schwierigkeiten heraus, zwischen inneren Zuständen (etwa Gefühlen) und äußerer Realität zu differenzieren, wobei Victor die eigenen Intentionen und inneren Zustände unzugänglich und fremd blieben (Modus der psychischen Äquivalenz). Das Fluktuieren der ohnehin beeinträchtigten Mentalisierungsfähigkeit hatte stressbedingt schließlich jene Schwelle erreicht, welche zum psychosozialen Zusammenbruch und dem Abbruch 
des Militärdienstes führte. Ein Jahr nach stationärer psychiatrischer und anschließend psychodynamischer Behandlung aufgrund dieser ersten durchlebten psychotischen Episode war Victor nun frei von akuten psychotischen Symptomen in medikamentöser Therapie mit Olanzapin. Dennoch gestaltete sich die Etablierung einer sicheren therapeutischen Beziehung als herausfordernd, vor allem aufgrund eines mutmaßlich unsichervermeidenden Bindungsstils. Scheinbar leicht zugänglich offenbarte Victor sich zunächst seinem Therapeuten, um später dessen inquisitives Vorgehen zu bemerken. Die von Brent durchgeführte und beschriebene therapeutische $\mathrm{Re}$ gulierung implizierte den Verzicht auf komplexe Übertragungsdeutungen und entspricht einem MBT-basierten Vorgehen mit Rücksicht auf die dem Patienten im situativen Beziehungskontext aktuell mögliche Mentalisierungsfähigkeit.

\section{Konklusion}

In den letzten Jahren wurden vermehrt psychotherapeutische Ansätze erforscht, welche insbesondere auf die Verbesserung von Metakognitionen und Mentalisierungsfähigkeit abzielten, um die sozialen Fähigkeiten zu verbessern. Einen mehrfach diskutierten Therapieansatz für Psychosen aus dem schizophrenen Formenkreis stellt eine adaptierte Form der mentalisierungsbasierten Therapie dar, welche ursprünglich für die Borderline-Persönlichkeitsstörung entwickelt wurde.

Die therapeutische Beziehung zeichnet sich aufseiten des Therapeuten durch eine interessierte, transparente und aktive Haltung aus. Der Fokus liegt auf der Aufrechterhaltung oder Wiederherstellung der Mentalisierungsfähigkeit über stressvolles Beziehungserleben. Hierbei ist eine Anpassung der Interventionen an die jeweilige Mentalisierungsfähigkeit in Abhängigkeit des Stressniveaus von großer Bedeutung, um einen Zusammenbruch der Mentalisierungsfähigkeit zu verhindern. Dafür ist es notwendig, auf komplexe, schwer verständliche Formulierungen zu verzichten. Psychosepatienten weisen häufig bereits bei basalen psychischen Aspekten (Identifikation eigener Gedan-

psychopraxis. neuropraxis 2020 - 23:175-179 https://doi.org/10.1007/s00739-020-00654-4 (c) Der/die Autor(en) 2020

\section{F. Richter $\cdot$ D. Steinmair $\cdot$ H. Löffler-Stastka \\ Mentalisierung bei Störungen aus dem schizophrenen Formenkreis. Fallorientierte Darstellung}

\section{Zusammenfassung}

Das Konzept der Mentalisierungsfähigkeit, etabliert ausgehend von der Theory of Mind (ToM), beinhaltet jene menschliche Fähigkeit, die eigenen mentalen Zustände (z. B. Gedanken, Gefühle, Motive) sowie entsprechend jene des Gegenübers, in einen Sinn- und Bedeutungszusammenhang zu bringen und aus diesem mehr oder weniger tiefen Verständnis hilfreiche Erklärungsmodelle für Verhalten zu generieren. Entwickeln und entfalten sich menschliche mentale Funktionen und Fähigkeiten im "Gegenseitigen-aufeinander-bezogen-Sein", so impliziert diese Weltsicht das Individuum als im Wesentlichen sozial determiniert. Leidensdruck infolge depressiver als auch schizophreniformer Störungen resultiert ganz wesentlich aus eingeschränkter Mentalisierungsfähigkeit, dies unabhängig von der unterschiedlichen Ätiologie der genannten Störungen.

Die folgenden Ausführungen legen dar, inwieweit Defizite in der Mentalisierung bei schizophreniformen Störungsbildern in Erscheinung treten. Diese Defizite sind gekennzeichnet durch eine mangelhafte Ausbildung von Metarepräsentationen und zeigen je nach Symptomatik verschiedene prämentalistische Verarbeitungsmodi und deren spezifische Ausformungen (Hypo-/Hypermentalisieren). An einer geschilderten Fallvignette wird dies verständlich.

\section{Schlüsselwörter}

Mentalisierung · Schizophrenie · Mentalisierungsbasierte Therapie - Psychologisches Selbst · Affekt-kognitive Prozesse

\section{Mentalisation in Disorders from the Schizophrenia Spectrum. Case-oriented Presentation}

\section{Abstract}

The concept of the ability to mentalise, established on the basis of the Theory of Mind (ToM), includes the human ability to bring one's own mental states (e.g. thoughts, feelings, motives) and those of the other person into a context of meaning and significance and to use this in many ways for deep understanding to generate helpful explanatory models for behaviour. If human mental functions and abilities develop and unfold in "mutual relation", this world view implies that the individual is essentially psycho-socially determined. Suffering from depressive as well as schizophreniform disorders results essentially from limited mental capacity, regardless of the varying aetiology of the disorders mentioned. The following observations show the extent to which deficits in mentalisation manifest in schizophreniform disorders. These deficits are characterised by deficient development of metarepresentations and, depending on the symptoms, show various pre-mentalistic processing methods and their specific forms (hypo-/hypermentalisation). This can be understood from the case vignette described.

\section{Keywords}

Mentalization · Schizophrenia · Mentalizationbased therapy - Psychological self · Affectcognitive interface ken und Gefühle) Schwierigkeiten auf, sodass tiefgründige Interpretationen von frühen Bindungserfahrungen vermieden werden sollten. Demnach sollten Interventionen einfach und klar formuliert sein, auf die mentalen Prozesse (insbesondere die Affekte) des Patienten im „Hier und Jetzt“ fokussiert sein sowie eine Verbindung zwischen Verhalten und dem eigenen Erleben gezogen werden. Positives Validieren von gelungenem Menta- lisieren ist hierbei förderlich. Der Therapeut zeigt sich aktiv mit seiner Mentalisierungsfähigkeit, um dem Patienten ein Modell zu bieten, woran er sich orientieren kann. 


\section{Fazit für die Praxis}

- Mentalisierungsfähigkeit und Bindungsfähigkeit sind untrennbar miteinander verbundene Konzepte.

- Mentalisierungskompetenz ist abhängig vom psychischen Funktionsniveau, zeigt störungsspezifisch typische Muster und Ausprägungen, fluktuiert situativ und kontextbezogen (bspw. Bindungsstress).

- Unabhängig von der zugrunde liegenden psychischen Störung stellt das Herstellen einer sicheren Beziehung die Basis für jegliche therapeutische Intervention dar.

- Die Qualität dieser therapeutischen Beziehung trägt entscheidend dazu bei, ob und wie sich dem Therapeuten prämentalistische Modi offenbaren und manifestieren können; die Identifikation der prämentalistischen Modi stellt den nun erforderlichen nächsten Schritt dar.

- Bei Psychosepatienten bieten sich eine Strukturierung der Sitzungen sowie verschiedene Übungen zur „Kultivierung" der Mentalisierungsfähigkeit an:

1. Mentalisierung der Persönlichkeit (,Wer bin ich?, Was macht mich aus?")

2. Imaginatives Mentalisieren (Foto interpretieren, Gedanken und Gefühle malen)

3. Mentalisieren interpersonaler Interaktionen und Beziehungen (Nachdenken über wichtige Bindungsperson, typische Interaktionen antizipieren)

- Schließlich kann eine Anpassung der mentalisierungsfördernden Interventionen an den jeweiligen Modus erfolgen.

- Bei einem Zusammenbruch der Mentalisierungsfähigkeit (bspw. Äquivalenzmodus) kann es notwendig sein, supportiv zu intervenieren, da alternative Perspektiven nicht mehr denkbar sind.

- Der Fokus sollte auf die mentalen Prozesse des Patienten gelegt werden (aufmerksames Zuhören, Spiegelung von Beobachtungen und Gedanken) und diese im Rahmen seiner aktuellen psychischen Möglichkei- ten anerkennen (Validieren), damit der Patient sich wieder sicher und verstanden fühlen kann.

- Sofern die Mentalisierungsfähigkeit wiederhergestellt ist, können verschiedene Perspektiven miteinbezogen werden oder auch innerhalb der Übertragung gearbeitet werden.

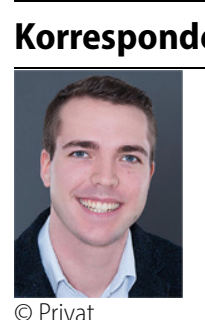

Felix Richter

Klinik für Psychoanalyse und

Psychotherapie, Medizinische

Universität Wien

Währinger Gürtel 18-20,

1090 Wien, Österreich

Felix-D-Richter@gmx.de

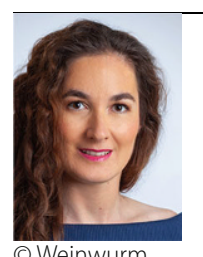

Dr. Dagmar Steinmair

Klinik für Psychoanalyse und

Psychotherapie, Medizinische Universität Wien

Währinger Gürtel 18-20,

1090 Wien, Österreich

2020

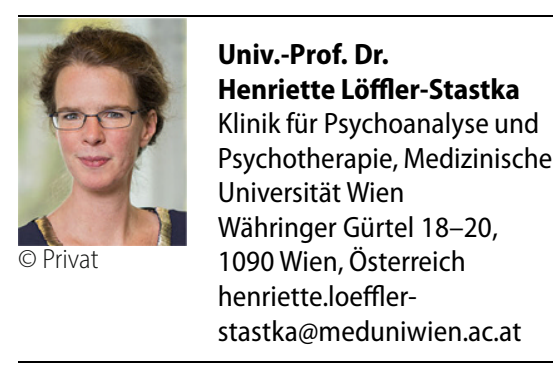

Funding. Open access funding provided by Medical University of Vienna.

\section{Einhaltung ethischer Richtlinien}

Interessenkonflikt. F. Richter, D. Steinmair und H. Löffler-Stastka geben an, dass kein Interessenkonflikt besteht.

Für diesen Beitrag wurden von den Autoren keine Studien an Menschen oder Tieren durchgeführt. Für die aufgeführten Studien gelten die jeweils dort angegebenen ethischen Richtlinien. Für Bildmaterial oder anderweitige Angaben innerhalb des Manuskripts, über die Patienten zu identifizieren sind, liegt von innen und/oder ihren gesetzlichen Vertretern eine schriftliche Einwilligung vor.

Open Access. Dieser Artikel wird unter der Creative Commons Namensnennung 4.0 International Lizenz veröffentlicht, welche die Nutzung, Vervielfältigung Bearbeitung, Verbreitung und Wiedergabe in jeglichem Medium und Format erlaubt, sofern Sie den/die ursprünglichen Autor(en) und die Quelle ordnungsgemäß nennen, einen Link zur Creative Commons Lizenz beifügen und angeben, ob Änderungen vorgenommen wurden.

Die in diesem Artikel enthaltenen Bilder und sonstiges Drittmaterial unterliegen ebenfalls der genannten Creative Commons Lizenz, sofern sich aus der Abbildungslegende nichts anderes ergibt. Sofern das betreffende Material nicht unter der genannten Creative Commons Lizenz steht und die betreffende Handlung nicht nach gesetzlichen Vorschriften erlaubt ist, ist für die oben aufgeführten Weiterverwendungen des Materials die Einwilligung des jeweiligen Rechteinhabers einzuholen.

Weitere Details zur Lizenz entnehmen Sie bitte der Lizenzinformation auf http://creativecommons.org/ licenses/by/4.0/deed.de.

Hinweis des Verlags. Der Verlag bleibt in Hinblick auf geografische Zuordnungen und Gebietsbezeich nungen in veröffentlichten Karten und Institutsadressen neutral. 